\title{
Optical Spin Orientation in Strained Superlattices
}

\author{
A.V. Subashiev, L.G. Gerchikov, and A.I. Ipatov \\ Department of Experimental Physics, State Polytechnical University, \\ 195251, Polytechnicheskaya 29, St.Petersburg, Russia
}

October 30, 2018

today

\begin{abstract}
Optical orientation in the strained semiconductor superlattices is investigated theoretically. The dependence of the features in spinpolarization spectra on the structure parameters is clarified. The value of polarization in the first polarization maximum in the SL structures is shown to grow with the splitting between the hh- and lh- states of the valence band, the joint strain and confinement effects on the hh1- lh1 splitting being strongly influenced by the tunneling in the barriers. In strained structures with high barriers for the holes initial polarization can exceed $95 \%$. Calculated polarization spectra are close to the experimental spectra of polarized electron emission.
\end{abstract}

Keywords: semiconductors, polarized electrons, strained layers, superlattices, photocathodes,

\section{Introduction}

Strained GaAs-based layers and superlattices are known to be most effective as photoemitters of spin-polarized electron beams which are required for highenergy experiments at many electron accelerator facilities [1, 2, 3]. Emitters 
of spin-polarized electrons will also be essential for future linear electron colliders (e.g. see Ref. [4] for the references).

High electron polarization is obtained by a spin optical orientation [5] which relies on the spin-orbit interaction in the valence band and selection rules for the interband optical transitions. Electron emission in vacuum at the near-band edge optical excitation is provided via activation of the structure surface by $\mathrm{Cs}(\mathrm{O})$ co-deposition to obtain a negative electron affinity.

In structures with compressively strained GaAs layer uniaxial deformation caused by the lattice mismatch of the working layer and a substrate results in a splitting of the 4 -fold degenerate $\Gamma_{8}$ valence band state into only 2-fold spin-degenerate states: the $\Gamma_{6}$ (heavy-hole) and the $\Gamma_{7}$ (light-hole) subband states, the heavy-hole band being moved up. The electrons excited by a circularly polarized light at the absorption edge from the heavy-hole subband with angular momentum $J=3 / 2$ populate only one electronic spin state of the conduction band. Therefore the electron polarization in the conduction band $|P|=100 \%$. At higher energies the light holes with $J=1 / 2$ start to contribute to the absorption via transitions to the second electron spin state with opposite direction of the electron spin orientation. This results in a decrease of the polarization with the excitation energy above the light-hole band excitation edge.

Unfortunately, since the critical thickness of coherent growth is of the order of $10 \mathrm{~nm}$, the photocathode strained layer structures are usually partly relaxed and thus have rather poor structural quality. Achievable valence band splittings in these structures are less than $50 \mathrm{meV}$ [6] which results in sizable polarization losses in the near band-gap excitation [7] via various processes that lead to the light-hole state contribution to the optical transition at the absorption edge.

In Quantum Well (QW) or Superlattice (SL) structures the valence band splitting is caused by the hole confinement in the QW layers. For these structures the theoretical limit for the initial polarization at the excitation is also $100 \%$. However, experimentally obtained values of the emitted electron polarization for unstrained SLs are typically less than $80 \%$. Since experimental studies of the electron depolarization in transport to the surface and emission in vacuum do not give more than $10 \%$ depolarization for high quality thinlayer structures [8], the initial electron polarization becomes an important issue.

The factors limiting polarization values in SL structures are the heavyhole-light-hole state mixing for finite hole momenta in the layer plane and 
also the hole scattering between minibands which lead to population of the second spin state. 9] To reduce the effects of these factors the enlarged value of the valence band splitting is again essential.

The main advantage of the strained SL structures is the potentially lager valence band splitting that results from the additive effects of hole confinement and strain. Besides, in the strained SLs higher total critical thicknesses can be achieved which ensures homogeneous strain and better structural quality of the working layers. As a result experimental values close to $P=90 \%$ were recently reported [10, 11. However, the band offset in the conduction band is usually larger than in the valence band, so hole confinement may be accompanied by strong electronic confinement. In this case the electrons are not mobile enough to reach the surface without some depolarization.

The emitted electron polarization depends on a number of structure parameters, including the band offsets, the transparency of the barrier layers for the electrons and holes etc. The flexibility of the SL structures allows one to adjust these parameters, though a specific structure design is needed. For this purpose it is important to have a qualitative comprehension of the main factors contributing to the variation of the polarization spectra with the SL parameters.

Former theoretical studies of the electron optical orientation were restricted to QW structures [12, 13] and SL structures with a fixed set of parameters 9, so that quantitative predictions for maximum $P$ values in SLs with different structure parameters were not reliable.

In this paper we concentrate on elucidation of the general aspects of optical orientation in unstrained and strained SLs and its dependence on the structure parameters, having in mind the structure optimization for photocathode applications. The role of the heterointerface effects on the polarization spectra is examined. The maximum polarization of electrons at the moment of excitation is discussed.

\section{Optical absorption and spin orientation}

\section{Effective Hamiltonian and band structure calculations}

The starting point of our calculations is finding the band structure and optical matrix elements for the circularly polarized light excitation and their dependence on the SL parameters. We use the envelope function approxi- 
mation in the multi-band Kane model including the conduction band $\Gamma_{6}$, the states of light and heavy holes of the valence band $\Gamma_{8}$ and also the states of the spin-orbit splitted band $\Gamma_{7}$. The electronic polarization defined by the concentration of the electrons with the spin parallel $\left(n_{\uparrow}\right)$ and antiparallel $\left(n_{\downarrow}\right)$ to the light propagation direction as

$$
P=\left(n_{\uparrow}-n_{\downarrow}\right) /\left(n_{\uparrow}+n_{\downarrow}\right),
$$

depends on the difference in the absorption for the two sets of states and therefore is more sensitive to the spectrum details than just an absorption coefficient.

For this reason we use the approach of Baraff and Gershoni 14, which is based on expansion of the envelope function in a Fourier series. The advantage of this is the possibility to trace easily the role of various factors in the electron optical orientation including the choice of the boundary conditions.

The wave function of the carrier in the SL structure is taken in the form

$$
\Psi(\mathbf{r})=\sum_{\nu=1}^{N} \psi^{\nu}(\mathbf{r}) u_{\nu}(\mathbf{r})
$$

where $\nu$ labels the periodic Bloch wave functions $u_{\nu}(\mathbf{r})$ of the included bands at the center of the Brillouin zone (which are supposed to be equal in both layers of the SL). The set of Bloch functions is taken as $|S \uparrow\rangle$ for the conduction band and $|X \uparrow\rangle,|Y \uparrow\rangle,|Z \uparrow\rangle$ for the valence band and also their time-reversed conjugates. In this basis the Hamiltonian is $8 \times 8$ matrix:

$$
H=\left(\begin{array}{cc}
G & \Gamma \\
-\Gamma^{*} & G^{*}
\end{array}\right)
$$

where $G$ and $\Gamma$ are both $4 \times 4$ matrixes defined in Ref. [15] and given explicitly in the Appendix. The band edge values $E_{c, v}$, the spin-orbital valence band splitting $\Delta$, Kane matrix elements $P$, and the band effective mass parameters $A, L, M, N$ are taken to change abruptly at the interfaces. Inside the $i$-th SL layer, $(i=1$ for a quantum well at $0<z<a$ and $i=2$ for a barrier at $a<z<a+b)$ these parameters are equal to their corresponding values for the bulk materials.

The SL translational symmetry leads to the Bloch type structure for the envelopes. Therefore, the states in the SL are characterized by the carrier 


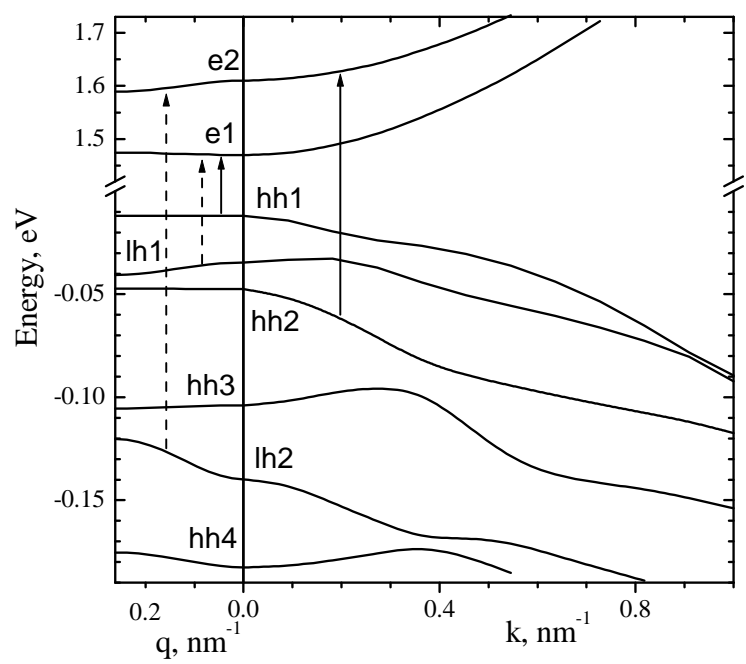

Figure 1: Miniband spectrum of $\mathrm{GaAs} / \mathrm{Al}_{0.4} \mathrm{Ga}_{0.6} \mathrm{As} 8 \times 4 \mathrm{~nm}$ superlattice and the major optical transitions to $|\uparrow\rangle$ (solid) and $|\downarrow\rangle$ states (dashed arrows) under circularly polarized light excitation.

wave vector parallel to interface $\mathbf{k}$ and by the component $q$ normal to interface, while the periodic part of the envelope can be found as a series expansion of plane waves

$$
\psi_{\mathbf{k}, q}^{(\nu)}(\mathbf{r})=\frac{1}{\sqrt{d}} e^{i \mathbf{k} \rho+i q z} \sum_{n} \exp \left(i \frac{2 \pi}{d} n z\right) A_{n, \nu}(\mathbf{k}, q)
$$

where $d=a+b$ is the SL period, and $n=0, \pm 1, \pm 2 \ldots$ The energy spectrum of the SL as well as the eigenstates of the problem are found from the solution of the matrix equation

$$
\sum_{n \nu}\langle m \mu|\hat{H}| n \nu\rangle A_{n \nu}(\mathbf{k}, q)=\varepsilon(\mathbf{k}, q) A_{m \mu}(\mathbf{k}, q) .
$$

The strain effects are readily incorporated by including an additional term, $H_{\text {strain }}$, in the effective Hamiltonian of each layer [14. The calculated miniband spectrum is shown in Fig. 1 for a (100)- oriented lattice-matched GaAs $/ \mathrm{Al}_{0.4} \mathrm{Ga}_{0.6}$ As SL structure assuming the electron barrier height $\Lambda_{c}=$ $0.313 \mathrm{eV}$ and the hole barrier $\Lambda_{v}=0.213 \mathrm{eV}$. In calculations we use the material parameters from Ref. [16] The thickness of the GaAs layer, $a=8$ $\mathrm{nm}$, is chosen to obtain several miniband states localized in the GaAs well 
layer both electrons and holes, and consequently several optical transitions contributing to the interband absorption spectrum near the edge. The width of the barriers are taken to be $b=4 \mathrm{~nm}$, so that the barriers are sufficiently transparent for electrons and light holes.

For the chosen well depth and thickness there are two electron (e), two light hole (lh) and five heavy hole (hh) confined quantum levels. The effects of tunnelling through the barriers are manifested in the electronic and lighthole miniband dispersion along the SL axis. We note here a small separation between the second heavy-hole (hh2) and the first light-hole (lh1) minibands at the center of the Brillouin zone that is typical for these structures since the effective mass ratio in GaAs is $m_{h h} / m_{l h} \approx 5$ and the lh1 miniband is shifted up towards the hh2 miniband due to the barrier transparency.

\section{Boundary conditions}

The choice of boundary conditions for the envelope functions $\psi^{\nu}$ is a central point for the approaches based on the effective mass model [17. In the transfer matrix approach [18, 19] a transfer matrix connecting the components of the envelope function and their derivatives on each side of an interface is postulated as a characteristic of the interface. An equivalent approach adopted here specifies variation of the effective mass Hamiltonian $\hat{H}$ of the SL given by Eq. (3) in the interface region [14] by inclusion additional interface terms 20, 21].

The step-like variation of $\hat{H}$ across the heterointerfaces results in a singular contribution to the matrix elements in Eq. (15) which can be written as a separate interface term in $\hat{H}_{s}$ dependent on (equivalent to) the choice of boundary conditions:

$$
\hat{H}_{s}=\mp i \delta\left(z-z_{n}\right)\left(\begin{array}{cc}
F_{1}-F_{2} & \mathbf{0} \\
\mathbf{0} & F_{1}^{*}-F_{2}^{*}
\end{array}\right) .
$$

Here $z_{n}$ corresponds to the plane of the $n$-th interface, the sign in the right side of Eq. (6) is different for the left and right interfaces of the QW, and $F_{i}$ in each layer is given by

$$
F=\left(\begin{array}{cccc}
A \hat{k}_{z} & 0 & 0 & \frac{i}{2} P \\
0 & M \hat{k}_{z} & 0 & \frac{1}{2} k_{x} N \\
0 & 0 & M \hat{k}_{z} & \frac{1}{2} k_{y} N \\
-\frac{i}{2} P & \frac{1}{2} k_{x} N & \frac{1}{2} k_{y} N & L \hat{k}_{z}
\end{array}\right) .
$$


Formally these type of terms arise from the action of $\hat{k}_{z}$ on the step-like dependent Kane parameters (see Appendix, Eq. (17)).

Here we use the symmetrization prescription of Ref. [14 for each matrix element in the SL Hamiltonian (see Appendix). However, as was pointed out and discussed in Refs. [17, 19, the induced symmetric form for each matrix element is excessive. The requirements that the SL Hamiltonian be Hermitian and have the symmetry of the bulk materials does not fix the order of operators $k_{z}=-i \partial / \partial z$ and band parameters in the Hamiltonian. Variation of this order results in an alteration of the matrix $F$

$$
\delta F=\left(\begin{array}{cccc}
0 & 0 & 0 & \frac{i \alpha}{2} P \\
0 & 0 & 0 & \frac{\beta}{2} k_{x} N \\
0 & 0 & 0 & \frac{\beta}{2} k_{y} N \\
\frac{i \alpha}{2} P & -\frac{\beta}{2} k_{x} N & -\frac{\beta}{2} k_{y} N & 0
\end{array}\right),
$$

where the parameters $\alpha$ and $\beta$ may vary in the interval $[-1,1]$ depending on the operator order which can not be rigorously justified.

The manifestations of $\alpha$ and $\beta$ terms in the SL spectra are quite different. Since the $\beta$ terms are proportional to the in-plane components of the wave vector $\mathbf{k}$ they do not affect the positions of the miniband centers, but slightly modify the dispersion of valence minibands. These corrections are of the order of the dispersion of the hh subbands reduced by the factor proportional to the probability to find a hole at the interface. In contrast, the $\alpha$ terms shift the position of the electron- and the lh- miniband centers and therefore may alter the splitting between the light and heavy hole minibands.

Note here that the effects of the boundary conditions for the alloy heterostructures are dependent of the choice of the Kane Hamiltonian parameters, namely, the evaluation of the first-order momentum matrix element between the $\Gamma_{1}$ and $\Gamma_{15}$ states, $|P|$, and the second-order momentum matrix elements and their difference in the well and barrier layers [17, 19]. For the structures with close values of these parameters in the layers [16] the effects of the interface in the SL miniband spectra are usually not large.

Then, it should be noted that the effective SL Hamiltonian (with a singular interface term in the form of Eq. (6) ) has the symmetry which follows from the symmetry of the bulk materials. As a result, for the (100)-oriented SL structure the reduction of translational symmetry does not lead to lightheavy hole transformations at the interfaces for holes moving along the SL axis [14. However, the actual point symmetry $C_{2 v}$ of the (001) interface of the III-V based SL structures allows the interface-induced mixing between 
the hh- and lh- states [20]. This coupling accounts for the interface-induced optical anisotropy in the interband transitions and the anisotropic exchange splitting of the $1 s$ heavy-hole exciton level in type II SLs 21] and also for the additional spin splitting of the states at $k \neq 0[22$.

The hh-lh mixing at the interfaces results from the mixing between $X$ and $Y$ orbital states of the valence band edge. This effect can be taken into account by adding to the valence band part of the Hamiltonian an extra surface term $H_{v, X-Y}$ 20]

$$
H_{v, X-Y}=\sum_{i}(-1)^{i} \frac{\hbar^{2} t_{x-y}}{m_{0} a_{0}}\left[\begin{array}{cc}
\left\{I_{X} I_{Y}\right\} & 0 \\
0 & \left\{I_{X} I_{Y}\right\}
\end{array}\right] \delta\left(z-z_{i}\right),
$$

where $a_{0}$ is the lattice constant, $t_{x-y}$ is a dimensionless mixing parameter, $z_{i}$ are the coordinates of the interfaces and $\left\{I_{X} I_{Y}\right\}$ is the symmetrized product of the matrixes for the angular momentum $I=1$,

$$
\left\{I_{X} I_{Y}\right\}=\frac{1}{2}\left[\begin{array}{ccc}
0 & -1 & 0 \\
-1 & 0 & 0 \\
0 & 0 & 0
\end{array}\right]
$$

which is invariant under $C_{2 v}$ group operations. The factors \pm 1 at the right and left interfaces of a chosen layer provide compensation of the asymmetries of the two surfaces of the layer in the average. As a result, at $\mathbf{k}, q=0$ only the hole miniband states with different parity are mixed. Then, the heterointerface hh-lh mixing term couples the hh and lh pairs of states with the angular momentum projections $+3 / 2$ and $-1 / 2$ and also $-1 / 2$ and $+3 / 2$. The mixing term of Eq. (9) should be transformed into a $8 \times 8$ matrix by the inclusion in the matrixes $\left\{I_{X} I_{Y}\right\}$ the first row and the first column with zero elements and then added to the SL Hamiltonian of Eq. (3).

An important consequence of the lowered symmetry of the interfaces is the spin splitting of the miniband states [22, 23]. Indeed, the SL Hamiltonian (3) has the inversion center and consequently the band energy spectrum obtained in Fig. 1 is spin degenerated. The interface terms (9) break this symmetry and induce the spin splitting linear in $k$ at $k=0$.

Besides, there is also a spin splitting caused by a bulk inversion asymmetry of each III-V semiconductor layers, originated from the so-called Dresselhaus terms in the bulk Hamiltonian. For the electron states both contributions are negligible due to a small admixture of the hole components to 
the conduction band envelopes. However, the spin splitting of the hole minibands originated from the interface terms (9) can be sizable and can exceed the contribution of the Dresselhaus terms [22].

The role of the interface originated asymmetry depends on the value of the mixing parameter $t$ which was estimated for some heterostructure interfaces in the tight binding approximation [20, 21, 24. Here (if not specified differently) we use for $t$ the interpolated values from Ref. 24]. The hole miniband spectra of GaAs/AlGaAs SL allowing $X-Y$ mixing with $t=0.6$ is shown for $\mathbf{k} \|$ (110) in the inset of Fig. 5. The splitting is most noticeable for the hh2 states and is highly anisotropic in the layer plane.

The effect of different boundary conditions and the consequences of the interface asymmetry for the polarization spectra are discussed below.

\section{Interband absorption and electron polarization}

The optical absorption coefficient is calculated as

$$
\alpha(\omega)=\frac{(2 \pi)^{2} e^{2} \omega}{\hbar c} \int \sum_{n, n^{\prime}}\left|M_{n^{\prime}, n}\right|^{2} \delta\left(\omega_{n n^{\prime}}(\mathbf{k}, q)-\omega\right) \frac{d \mathbf{k} d q}{(2 \pi)^{3}},
$$

where $M_{n^{\prime}, n}=\left\langle\Psi_{n^{\prime}, \mathbf{k}, \mathbf{q}}|\mathbf{e r}| \Psi_{n, \mathbf{k}, q}\right\rangle$ is the dipole matrix element of the optical transition between the $n$ and $n^{\prime}$ minibands, e is the photon polarization vector located in the interface plane, and $\hbar \omega_{n n^{\prime}}(\mathbf{k}, q)=\varepsilon_{n^{\prime}}(\mathbf{k}, q)-\varepsilon_{n}(\mathbf{k}, q)$ is the excitation energy. The sum in Eq. (11) is performed over all $n$-th occupied and $n^{\prime}$-th vacant subbands [25].

In order to calculate the spin polarization of generated electrons using Eq.(11) for the circularly polarized light propagating along the SL axis (i.e., for $\left.\mathbf{e}=\left(\mathbf{e}_{x} \pm i \mathbf{e}_{y}\right) / 2\right)$ one should distinguish the final electron states with "Up" $(\uparrow)$ and "Down" $(\downarrow)$ spin projections on this axis. We will introduce two absorption coefficients $\alpha_{\uparrow}$ and $\alpha_{\downarrow}$ for the optical transitions to the $|\uparrow\rangle$ and $|\downarrow\rangle$ final electronic states, respectively, and assume that $n_{\uparrow, \downarrow} \propto \alpha_{\uparrow, \downarrow}$.

Then the absorption coefficients, $\alpha_{\uparrow}$ and $\alpha_{\downarrow}$, are calculated via the density matrix $\hat{\rho}$ for the optical transitions and the projection operators $\hat{P}_{\uparrow}$ and $\hat{P}_{\downarrow}$ as

$$
\alpha_{\uparrow, \downarrow}=S p\left\{\hat{\rho} \hat{P}_{\uparrow, \downarrow}\right\}
$$

The trace here implies also the integration over $\mathbf{k}$ and $q$ in addition to the summation over the electron minibands. The density matrix for the optical 
transitions is defined as

$$
\begin{gathered}
\hat{\rho}_{n_{2} n_{1}}(\mathbf{k}, q)= \\
\frac{(2 \pi e)^{2} \omega}{\hbar c} \sum_{n} M_{n, n_{2}}^{*}(\mathbf{k}, q) M_{n, n_{1}}(\mathbf{k}, q) \delta\left(\omega_{n n_{1}}(\mathbf{k}, q)-\omega\right) .
\end{gathered}
$$

Here indexes $n_{2}$ and $n_{1}$ correspond to the twofold degenerate subbband states. The matrix elements of the projection operators are equal to

$$
\left\langle\Psi_{n^{\prime}, \mathbf{k}, q}\left|\hat{P}_{\uparrow, \downarrow}\right| \Psi_{n, \mathbf{k}, q}\right\rangle=C_{\nu} \sum_{i} A_{i \nu}^{*}\left(n^{\prime}, \mathbf{k}, q\right) A_{i \nu}(n, \mathbf{k}, q),
$$

where $\nu=1$ for $\hat{P}_{\uparrow}$ and $\nu=5$ for $\hat{P}_{\downarrow} ; C_{\nu}=\left(\sum_{n, i}\left|A_{i \nu}(n, \mathbf{k}, q)\right|^{2}\right)^{-1}$. The optical matrix elements for the interband transitions in Eqs. (1113) are calculated straightforwardly as

$$
M_{n^{\prime}, n}(\mathbf{k}, q)=\left\langle\Psi_{n^{\prime}, \mathbf{k}, q}\left|\mathbf{e} \nabla_{\mathbf{k}} \hat{H}\right| \Psi_{n, \mathbf{k}, q}\right\rangle / \hbar \omega_{n n^{\prime}}(\mathbf{k}, q),
$$

though a singular interface contribution is also present (e.g. see Ref. [26]). Squared matrix elements for the transitions to the $|\uparrow\rangle$ and $|\downarrow\rangle$ subband states are shown in Fig. 2 as a function of wave vector for the same structure as in Fig. 1.

Strong dispersion of the matrix elements for the interband transitions under excitation by circularly polarized light originates from the strict selection rules for the optical transitions at the boundaries of the Brillouin zone $\mathbf{k}=0$ and $q=0, \pi / d$, connected with the miniband spin and parity.

Since the Bloch functions of the conduction band $(|S \uparrow, \downarrow\rangle)$ and the valence band (e.g. $|X \uparrow, \downarrow\rangle$ ) have the opposite parity, the optical matrix elements, $M_{n^{\prime}, n}$, are non-zero for the transitions between minibands with the same envelope parity (e.g. hh1 $\rightarrow$ e1). Among the allowed transitions the largest absolute value of $M_{n^{\prime}, n}$ has transitions between minibands with equal numbers, e.g. hh1 $\rightarrow$ e1 and lh1 $\rightarrow$ e1, with the largest overlap of the envelopes.

Besides the spatial dependence, the spin structure of the envelopes is also important. The spin-polarized electrons are generated by circularly polarized light with photon angular momentum $I=1$. For an angular momentum projection of +1 , the electron states with $|\uparrow\rangle$ spin projection on the SL axes (opposite to the light propagation direction in the reflection geometry) will only be populated at $\mathbf{k}=0$ due to the transitions from the hh states with the total angular momentum projection $+3 / 2$. All the other optical transitions 


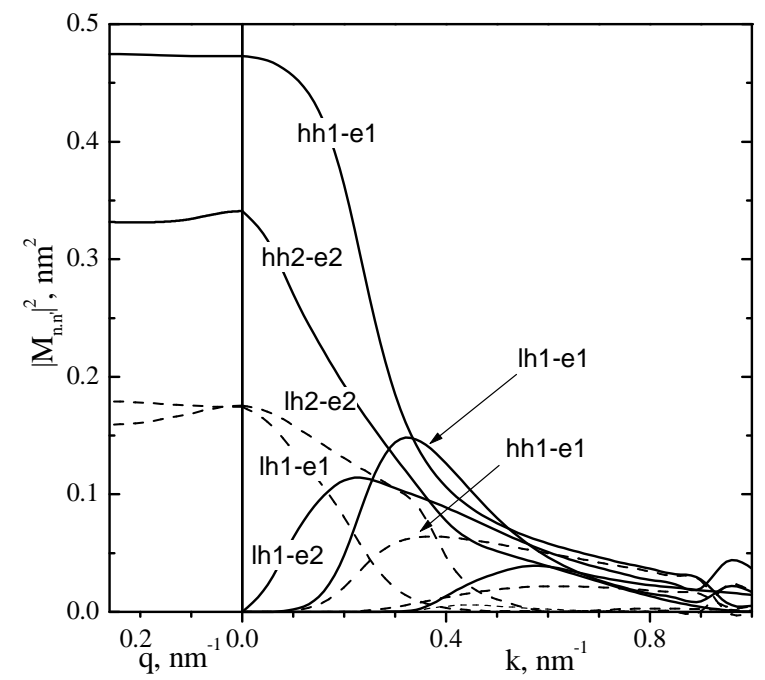

Figure 2: Squared optical matrix elements for the transitions to spin-up (solid) and spin down states (dashed lines) as a function of the wave vector in the plane of the interface $(\mathbf{k} \|(100))$ and normal to the SL interfaces $(q)$ for the same structure as in Fig. 1.

are forbidden by the conservation of angular momentum. Similarly, the $|\downarrow\rangle$ states are populated by the transitions from the light hole states with the total angular momentum projection of $+1 / 2$. In Fig. 2 we show the squared matrix elements $\left|M_{n^{\prime}, n}\right|^{2}$ for main optical transitions, solid and dashed lines correspond to the transitions in the $|\uparrow\rangle$ and $|\downarrow\rangle$ states, respectively.

These selection rules are released at $\mathbf{k} \neq 0$ due to the mixing of the heavy and light hole components of different parity and spin projections. The resulting dispersion of matrix elements in the interface plane for $\mathbf{k} \|$ (100) is shown in Fig. 2. With the growth of the in-plane momentum, $k$, the optical matrix elements decrease for the allowed transitions and increase for the forbidden transitions. For example, one can see a rather rapid growth of the matrix element of the $\ln 1 \rightarrow \mathrm{e} 2$ transition forbidden by parity at $\mathbf{k}=0$. It originates from the mixture of the lh1 miniband with the close hh2 states (see Fig. 1). Consequently, the oscillator strength of the $l h 1 \rightarrow \mathrm{e} 2$ transition decreases. 


\section{Calculation results}

\section{Unstrained structures}

Up and down contributions to the photoabsorption coefficient (dashed line and dot-dashed line) and also the total absorption spectrum (solid line) of the unstrained GaAs/AlGaAs SL are shown in Fig. 3 (a). To give deeper insight into the optical spectrum formation the partial contributions of the most important optical transitions are also shown by thin solid lines.

In a manner similar to the density of states in multi-QW structures, the optical absorption spectrum has a step function character, the most noticeable steps coming from transitions that have large enough optical matrix elements at their absorption edge. The steep edges in the polarized light absorption hh1-e1 and hh2-e2 are responsible for the steps in the generation of $|\uparrow\rangle$ polarized electrons, while the transitions lh1-e1 and lh2-e2 provide the steps in the $|\downarrow\rangle$ electron excitation.

The transition e2-hh2 needs more comment. According to Fig. 3 (a) the contribution of the hh2 - e2 transition itself provides only half of the second step in up-polarized absorbtion. The other half is the contribution of the e2 - lh1 transition which is forbidden by parity at the absorption edge and therefore should not contribute at all. However, in the SL structure under consideration the edges of hh2 and lh1 minibands are very close. This fact leads to a strong mixing between these states resulting in the sharing of the oscillator strength between the hh2-e2 and lh1-e1 transitions.

While the overall shape of the polarized absorption spectrum is mostly determined by the optical transitions between the electron and hole subbands with equal quantum numbers, similarly to the vertical optical transitions in the bulk, we note that these transitions are responsible only for the sharp step-like edges in the optical spectrum, their positions and magnitudes. The contribution of these transitions decreases rapidly away from their edges. This feature results from the decrease of the optical matrix elements with the growth of the electron momentum in the heterostructure plane [9] (see Fig. 2). The rather flat steps in the absorption come from the combined contribution of different optical transitions.

For example, the allowed lh1-e1 transition gives rise to the first step edge for the down-polarized absorption, though an almost constant value of generation rate at larger photon energies results from the additional contribution of the hh1-e1 transition. Note here that the hh1-e1 transition does not con- 

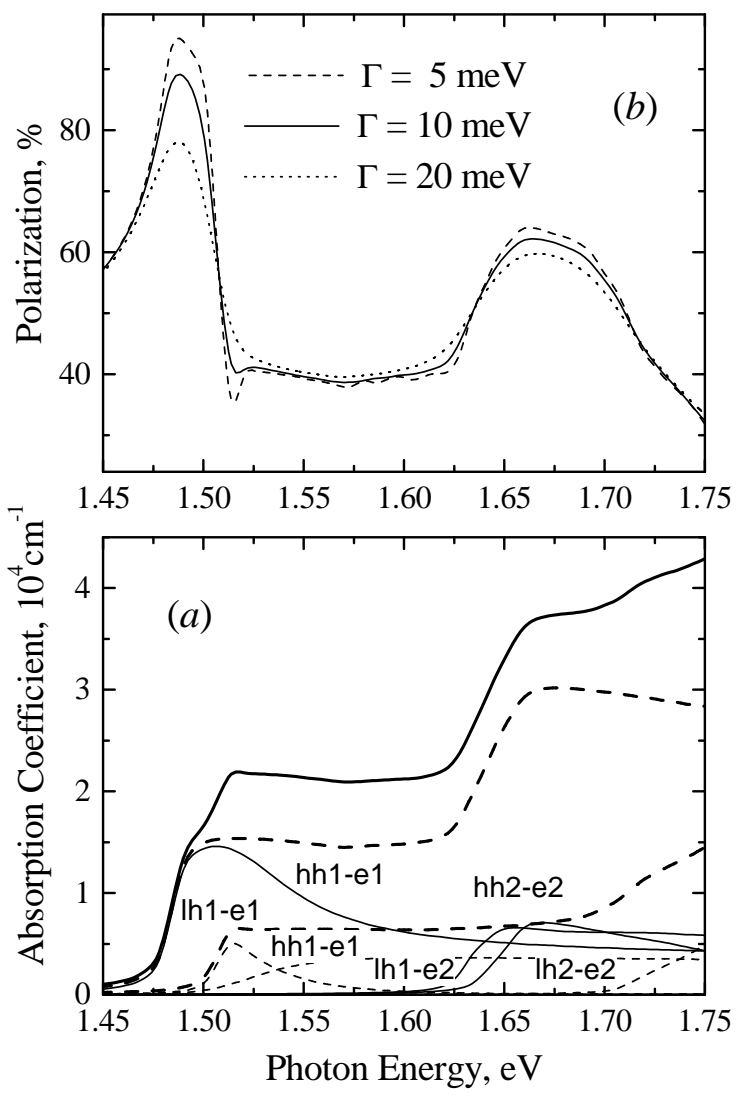

Figure 3: Calculated optical absorbtion of $\mathrm{GaAs} / \mathrm{Al}_{0.4} \mathrm{Ga}_{0.6}$ As superlattice with $a=8 \mathrm{~nm}, b=4 \mathrm{~nm}$ and $\gamma=7 \mathrm{meV}$ (a) and polarization spectrum of this superlattice (b); polarization spectra calculated for smaller $(\gamma=5 \mathrm{meV})$ and larger $(\gamma=20 \mathrm{meV})$ level width broadening are shown by dashed and dotted lines. 
tribute at its edge to the generation of down polarized electrons at all.

The step structure of the absorption spectrum determines the excitation energy dependence of the electron polarization at the excitation shown in Fig. 3 (b). In the interval between the left step edges in up and down absorption rates the electron polarization is close to $100 \%$. Similarly to the strain-induced electron polarization, this effect arises from the splitting between the heavy and light hole subbands in the SL structure. The width of the first polarization peak depends on the energy separation between hh1 and lh1 minibands. However, in addition to the first peak, there are also the repetitions of the first polarization peak associated with the higher electronic minibands. The strongest is the second polarization peak (see Fig. 3 (b)), which originates from the optical transitions to the second electron miniband, e2. Its position, shape and the maximum polarization value are dependent on the dispersion of the e2- miniband and the resulting optical density of states while its width is determined by the distance between the hh2 and lh2 levels. Therefore, the polarization magnitude in the second polarization peak can exceed $P=50 \%$ (the maximum value for bulk crystal). Then polarization decreases with the growth of the excitation energy and total absorption value. This decrease becomes very rapid when excitation energy is enough to induce transitions from the spin-orbital split valence band.

Note, that the shape of the polarization curve and the maximum polarization are sensitive to the width of the miniband levels and to the smearing of the interband absorption edges. In our calculations we use the Lorentzian broadening of the quantum levels. The actual level width in the real heterostructure originates from the interaction of the carriers with phonons and impurities and from fluctuations of layer composition and thickness and possibly other heterostructure defects. For the best SL structures absorbtion edge smearing was reported to be in the range $\gamma=7-12 \mathrm{meV}$ at room temperature [27, depending on doping and structural quality. Within the present work we use the level width as a parameter.

In Fig. 3 (a) the absorption and electron polarization spectra calculated for $\gamma=10 \mathrm{meV}$ are shown by the solid lines. The variation of the polarization spectrum with the level width is shown in Fig. 3 (b) by the dashed (for $\gamma=$ $5 \mathrm{meV})$ and dotted $(\gamma=20 \mathrm{meV})$ lines. For larger values of $\gamma$ along with the smearing of the absorption step edges, the polarization peaks become more narrow and smaller in magnitude. The long tail in the lh - e1 optical transitions reduces the amplitude of the main polarization maximum. Thus, the broadening of the SL energy spectrum sets the limit for the highest possible 
initial photoelectron polarization. A destructive effect of the broadening is reduced in the case of a wider polarization peak, i.e. in the case of a larger hh - lh miniband splitting.

In Fig. 4 the SL polarization spectra for different barrier thickness $b=$ 1.5, 2.5 and $4 \mathrm{~nm}$ are depicted to show the influence of tunneling through the SL barriers. For the barriers thicker than $4 \mathrm{~nm}$ the polarization spectra are found to be similar to that of the MQW structure with nontransparent barriers. For thinner barriers the dispersion of the minibands along the SL axis starts to result in a narrowing of the polarization peaks along with the decrease of the polarization. This decrease is more pronounced on the right

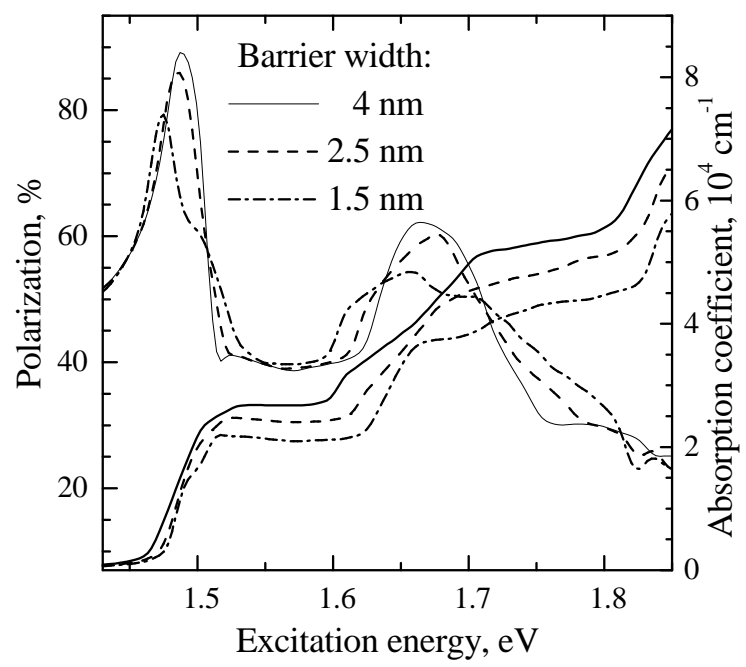

Figure 4: Polarization and absorption spectra for the GaAs/ $\mathrm{Al}_{0.4} \mathrm{Ga}_{0.6}$ As SL structures with $a=8 \mathrm{~nm}$ and different barrier layer thicknesses; $\gamma=10 \mathrm{meV}$.

side of the first polarization maximum showing the effect of the transitions from the lh minibands. The tunnelling probability of light holes is larger than that for heavy holes which results in a larger dispersion along the SL axis and therefore a larger spread of their input to the polarization spectrum. This dispersion is also manifested in the smearing of the high-energy features in the absorption spectra of Fig. 4. In the limit of extremely narrow barriers $b \leq 1 \mathrm{~nm}$, the peak structure is damped while the SL polarization spectrum is transformed into the spectrum of the bulk material.

It should be noted that the width of the first polarization peak due to the splitting of the lh1 and hh1 minibands in unstrained SL structures with 
thick QW layers can be estimated as $\Delta E_{\mathrm{hh} 1, \mathrm{lh} 1} \approx \hbar^{2} \pi^{2} / a^{2}\left(m_{\mathrm{lh}}^{-1}-m_{\mathrm{hh}}^{-1}\right)$ (i.e. the value for an isolated deep QW) and is enlarged in the structures with narrowed wells. However the rapid growth of the splitting $\Delta E_{\mathrm{hh} 1, \mathrm{lh} 1} \propto a^{-2}$ with a decrease of $a$ presents itself only when $E_{l h 1} \ll \Lambda_{v}$ where $E_{l h 1}$ is the lh1 miniband energy at $\mathbf{k}, q=0$. Then the splitting saturates and decreases, mainly due to the effects of lh tunnelling in the barriers.

Actually, the maximum valence band splitting is obtained for the structures where $E_{\mathbf{l h} \mathbf{1}}$ is substantially smaller than $\Lambda_{\mathrm{v}}$. For the GaAs $/ \mathrm{Al}_{0.4} \mathrm{Ga}_{0.6} \mathrm{As}$ SL it turns out to be about four times smaller than $\Lambda_{\mathrm{v}}$. The maximum splitting value $43 \mathrm{meV}$ is achieved at the well width $a=2.6 \mathrm{~nm}$. The calculated polarization spectrum of this SL with $a=2.6 \mathrm{~nm}, b=3.6 \mathrm{~nm}$ is presented in Fig. 5 showing the growth of the maximum polarization value to $P \approx$ $93 \%$ at the maximum hh1-lh1 splitting. Finally, the splitting is reduced in

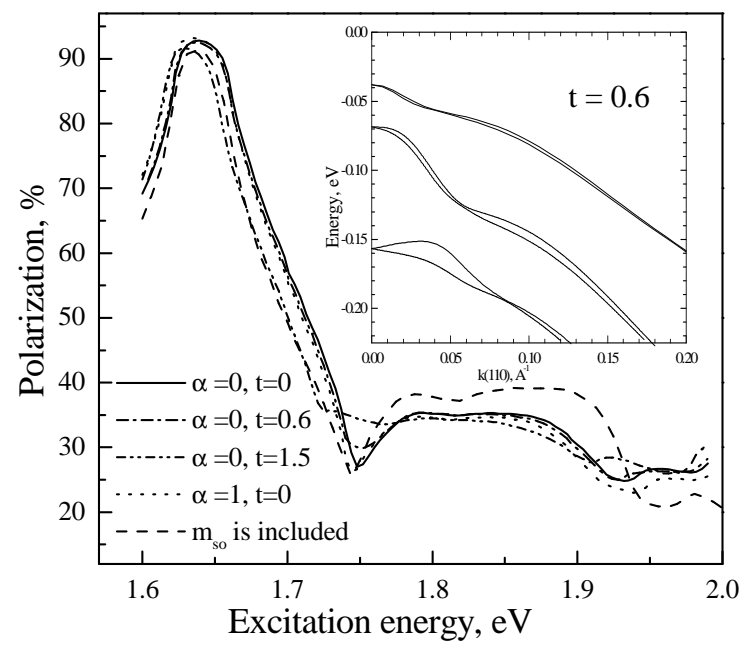

Figure 5: The influence of the interface hh-lh band mixing ( $t$ being the mixing parameter) on the polarization spectrum of InGaAs/AlGaAs structure for two choices of the boundary conditions $(\alpha)$. Dashed line shows results of the calculations with interband matrix element $P$ determined with the use of effective mass of the spin-orbit split valence band; $\gamma=10 \mathrm{meV}$; the hole miniband spectra is shown in the inset.

structures with thin valence band barriers, transparent for light holes, while in structures with high and low-transparent barriers in the conduction band the electrons are not mobile enough. This makes unstrained structures with 
typically higher barriers for electrons less favorable for polarized photoemission.

\section{Interface effects}

As stated above, the choice of the boundary conditions determined by the behavior of the SL Hamiltonian in the heterointerface regions, can result in variations in the calculated absorption and polarization spectra and in the maximum polarization value. These variations are illustrated by Fig. 5 in which the polarization spectra for 2.8 x $3.6 \mathrm{~nm}$ GaAs/ $\mathrm{Al}_{0.4} \mathrm{Ga}_{0.6} \mathrm{As} \mathrm{SL}$ with different boundary conditions are depicted.

The spectra obtained for various assumptions about $\alpha$ values are rather close, the deviations between the $\alpha=0$ and $\alpha=1$ cases being essentially due to a small shift of the electron quantum levels. The influence of the $\beta$ terms on polarization spectra is found to be negligible. Since the boundary conditions can not be derived unambiguously within the effective mass approximation the deviations in the spectra can be regarded as an error bar for the approach employed and in what follows we take $\alpha=\beta=0$.

The minor effects of the boundary conditions on the calculated spectra result from the fact that the first-order momentum matrix elements $P$ do not differ much in the SL layers. The parameters of the effective mass Hamiltonian (3)), including $P$, can be defined in terms of the experimental band gap, spin-orbital splitting, effective masses, and the $E_{\mathbf{p}}$ values, $E_{\mathbf{p}}=2 m_{0} P^{2} / \hbar^{2}$, evaluated from the experimental data on the nonparabolicity of the conduction band spectrum [16. In our calculations we use for the Hamiltonian parameters interpolation scheme and values recommended in Ref. [16].

The alternative is to use the data on the spin-orbit split-off band effective mass but to ignore the $E_{\mathbf{p}}$ data, see a dashed-line spectrum in Fig. 5 . In this

case the calculation results are considerably more sensitive to the choice of the boundary conditions which makes the conclusions that could be drawn from them less reliable.

Then, different interface effects originate from the additional term (9) in the SL Hamiltonian which mixes hh and lh states. In Fig. 5 we show by the dotted line the polarization spectrum of the $\mathrm{GaAs} / \mathrm{Al}_{0.4} \mathrm{Ga}_{0.4} \mathrm{As}$ SL allowing for the interface hh-lh mixing. The value of mixing parameter $t=0.6$ is taken by an interpolation of the values given in Ref. 24]. Note here that since the term couple $\mathrm{lh}$ and hh states with the opposite parity, the major effect comes from the hh2 and lh1 miniband interaction. While the mixing 
is neglected, the repulsion between closely situated hh- and lh- minibands at $k \neq 0$ leads to a large or even negative effective mass of the upper subband manifested as an additional feature in the absorbtion and a dip in polarization spectra. The repulsion between lh1 and hh2 minibands at the zone center increases the interlevel distance and therefore smears the described feature in the polarization spectrum.

The linear in $k$ anisotropic splitting of the hole minibands (seen in the inset of Fig. 5) being integrated over the $k$ directions does not show itself in polarization spectra.

The influence of the interface term of Eq. (9) on the main polarization maximum is of minor importance for $t \leq 1$. The admixture of the lh2 component to the hh1 wave function does not affect the amplitude of the polarization peak since it does not contribute to the optical transition to the e1 electron state with an opposite parity, the resulting shift of the hh1 miniband being not larger than a few meV.

\section{Strained structures}

In strained SL structures the lattice mismatch between SL layers and a substrate is aimed to results in an additional hh- lh- miniband splitting. Several types of structures favorable for photoemission with (i) strained SL wells [11, 28] (ii) stained barriers [29, 30] and (iii) employing strain compensation [31] are designed and tested. We now discuss how polarization spectra evolve with strain for realistic SL structures.

(i) Strained wells. First, we consider polarization spectra of a (100)oriented $\mathrm{GaP}_{0.4} \mathrm{As}_{0.6} 4 \mathrm{x} 4 \mathrm{~nm}$ SL structure with strained well layers. The band offsets in this SL are comparable to these in the $\mathrm{Al}_{0.4} \mathrm{Ga}_{0.6}$ As SL structure but the lattice constant in the barriers is smaller than that in the wells. The resulting deformation of the SL with finite total thickness strongly depends upon the substrate lattice constant. In Fig. 6 we show the optical absorption and polarization spectra for $\mathrm{GaAs} / \mathrm{GaP}_{0.4} \mathrm{As}_{0.6} \mathrm{SL}$ calculated for the structure grown on $\mathrm{GaP}_{\mathrm{x}} \mathrm{As}_{1-\mathrm{x}}$ pseudo-substrates with three different concentrations of P. The thickness of the SL structure is assumed to be much less than that of the substrate so that the resulting lattice constant of the layers in the layer plane accommodates completely to the substrate. The GaAs QW layers are stressed in the layer plane so that the hh- subband is pushed up while the lh- subband moves down. The strain in the QW layers and therefore the valence splitting in QW increases with the growth of the 


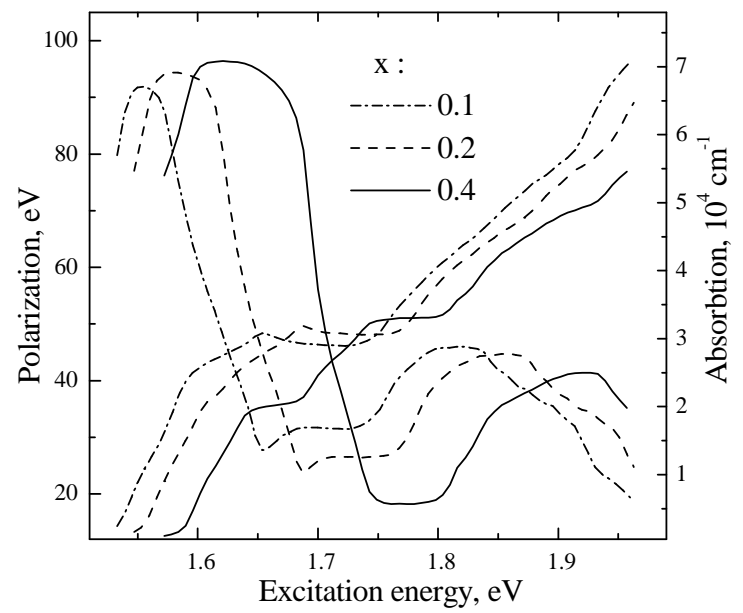

Figure 6: Polarization and absorption spectra of strained well GaAs $/ \mathrm{GaAs}_{0.6} \mathrm{P}_{0.4}$ SL structure for different $\mathrm{P}$ concentrations, $x$, in the substrate; $\gamma=10 \mathrm{meV}$.

$\mathrm{P}$ concentration in the substrate. For the substrate compositions considered, $\mathrm{x}=0.1,0.2,0.4$, the hh-lh valence band splitting is equal to 23,44 and 82 $\mathrm{meV}$, respectively. Since the barriers are tensile strained in the case of $\mathrm{x}=0.1$, 0.2 and unstrained at $\mathrm{x}=0.4$, the shifts of the barrier lh- and hh- band edges due to the barrier deformation has an opposite sign. As a result the valence band offset for the light holes $\left(\Lambda_{l h}=0.18 \mathrm{eV}\right)$ becomes smaller than for the heavy holes $\left(\Lambda_{h h}=0.28 \mathrm{eV}\right)$, their values being almost independent upon the substrate composition.

The decrease of the well depth for the light holes reduces the difference between the confinement energies of hh1 and lh1 miniband states at $\mathbf{k}, q$ $=0$ to about $20 \mathrm{meV}$ which is almost two times smaller than that in the unstrained GaAs $/ \mathrm{Al}_{0.4} \mathrm{Ga}_{0.6} \mathrm{As}$ SL with the same layer width. The total energy splitting between hh1 and lh1 states, being the sum of the confinement energy difference and the QW valence band splitting, equals to 45, 65 and $99 \mathrm{meV}$ (for $\mathrm{x}=0.1,0.2$ and 0.4 , respectively).

Fig. 6 shows the evolution of the spectra with the increase of strain in the well layers. It can be seen that the steps in absorption spectra and the main polarization peak in the strained SL become wider as a result of the larger hh1 and lh1 miniband separation due to join effects of the confinement and strain. The maximum polarization value rises to $\approx 97 \%$ for a SL with 
stressed wells and unstrained barriers (i.e. for $x=0.4$ ), while the minimum in the polarization spectra becomes more deep and wider.

(ii) Strained barriers. Choice of the barrier and well compositions allows one to design SL structures with strained barriers in which the valence band splitting increases the barrier height for light holes and decreases it for the heavy holes. As a result the difference between the lh- and hh- state energies is enlarged.

As an example we consider the polarization spectra for a GaAs based structure with ternary $\mathrm{Al}_{\mathrm{x}} \operatorname{In}_{\mathrm{y}} \mathrm{Ga}_{1-\mathrm{x}-\mathrm{y}}$ As alloy barriers grown on a GaAs substrate. The lattice constant of the barrier alloy is larger than in GaAs, which provides the needed sign of the valence band splitting in the barriers, while the QW layers remain unstrained. The barrier alloy composition $\mathrm{Al}_{0.21}$ $\mathrm{In}_{0.2} \mathrm{Ga}_{0.6} \mathrm{As}$ is chosen to achieve a close to zero value of the conduction band offset and therefore a high vertical electron mobility appropriate for photoemission [10, 29]. In Fig. 7 the optical absorption and polarization

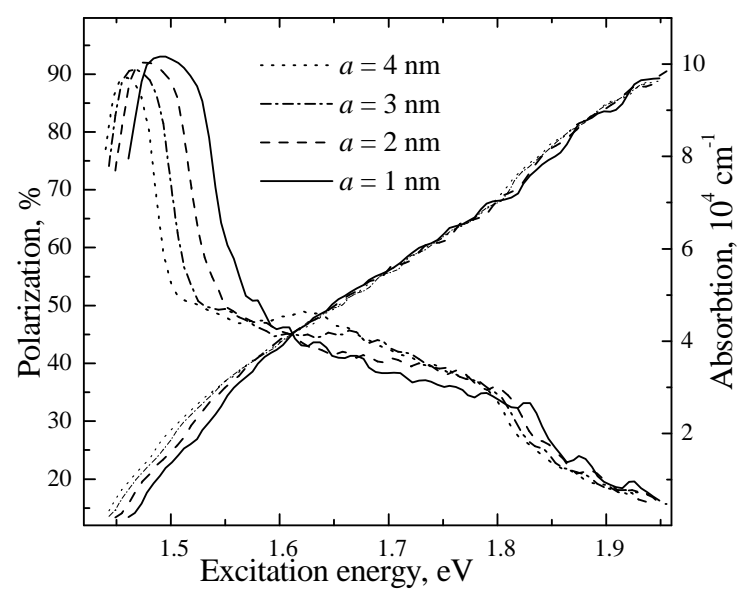

Figure 7: Polarization and absorption spectra of GaAs/ $\mathrm{Al}_{0.21} \operatorname{In}_{0.2} \mathrm{Ga}_{0.69}$ As SL with strained barriers and close to zero conduction band offset with $b=$ $4 \mathrm{~nm}$ and different QW thicknesses, $\gamma=10 \mathrm{meV}$.

spectra calculated for this type of SL with $4 \mathrm{~nm}$ barriers and different well thicknesses are shown. The valence band offsets for hh- and lh- subbands are equal to 62 and $140 \mathrm{meV}$, respectively. Consequently the energy difference between the hh1 and lh1 miniband states becomes larger than that in the GaAs QW SL with unstrained 100 meV- hight barriers though the hh1- 
lh1 splitting remains smaller than the deformation splitting in the barrier layers. Small conduction band offset results in the complete smearing of the features in the absorbtion spectra. A high polarization $(\approx 92 \%)$ is obtained in the structures with considerably thinner $(\leq 2 \mathrm{~nm})$ QW layers with hh1-lh1 splitting of about $64 \mathrm{meV}$.

(iii) Strain compensation. The thickness of a strained SL necessary for photoemission exceeds by an order of magnitude the critical thickness for the coherent layer growth, which leads to the strain relaxation, defective structures and polarization losses. The use of strain compensation, whereby the composition of the SL barrier layers is chosen to have opposite (tensile) strain from that of the quantum well layers is proposed to overcome limitations on the overall thickness of the SL structure [31.

The calculated spectra of strain compensated $\operatorname{In}_{0.2} \mathrm{Ga}_{0.8} \mathrm{As} / \mathrm{GaAs}_{0.65} \mathrm{P}_{0.35}$ Sl structures on GaAs substrate with different well width are shown in Fig. 8. In this structure the valence band splitting in the well and barrier layers have a close magnitude $\approx 78 \mathrm{meV}$ but differ by the sign. Consequently the well depth for the heavy holes is five times larger than for the light holes. Thus the heavy hole confinement energy has much stronger dependence on the well thickness then the lh1 energy and therefore the hh-lh splitting is mainly determined by the position of the hh1 miniband. The maximum polarization is obtained for the structures with thicker QW ( $a \geq 4 \mathrm{~nm}$ ) layers, with hh1-lh1 splitting $(\geq 60 \mathrm{meV})$ remaining lower than splitting caused by deformation in QW layers. It should be noted that the splitting is considerably reduced in the structures with thin QW layers due to more rapid growth of the hh1 state energy. Note here that the resulting valence band splitting and the electron initial polarization in the strain compensated structures are lowered (compared to strained well structures) due to the lowered barriers for the light holes formed by the tensile-strained layers.

A drawback of the strained well and strain compensated structures is typically the high barriers for electrons having reduced transparency, a disadvantage which is absent in strained barrier SL with zero conduction band offsets.

\section{Discussion}

Calculated dependencies of the electronic polarization of the excitation energy are close to the experimental spectra of polarized electron emission for 


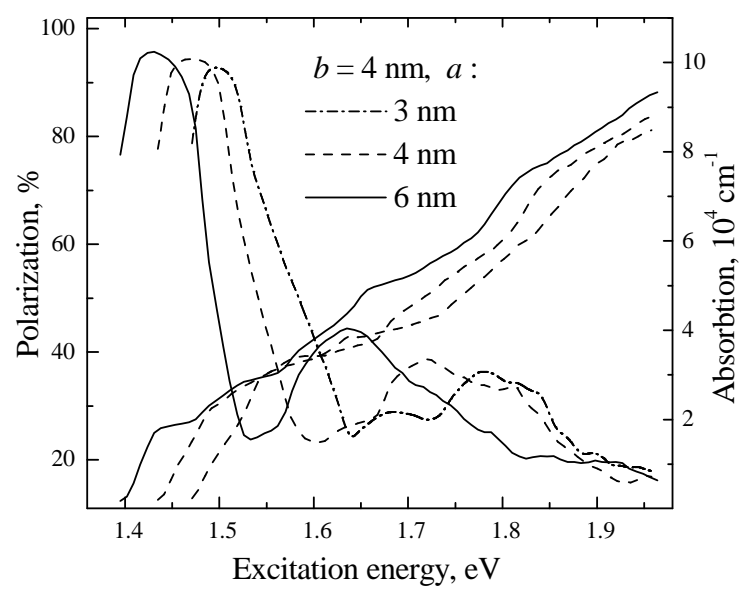

Figure 8: Polarization and absorption spectra of strain-compensated $\mathrm{Al}_{0.1}$ $\mathrm{In}_{0.2} \mathrm{Ga}_{0.7} \mathrm{As} / \mathrm{GaAs}_{0.7} \mathrm{P}_{0.3} \mathrm{SL}$ with $b=4 \mathrm{~nm}$ and different $\mathrm{QW}$ thicknesses; $\gamma=10 \mathrm{meV}$.

investigated structures [10, 11, 28, 30]. The value of polarization in the first polarization maximum in the SL structures under consideration ranges from 87 to $95 \%$ for the broadening of the absorption edge $\gamma=10 \mathrm{meV}$ taken here, and is noticeably growing with the splitting between the hh- and lh- states of the valence band. We have shown that the strain and confinement effects on the hh1- lh1 splittings do not simply add up mainly because the lh confinement energy is strongly influenced by the tunnelling in the barriers, the range of optimal layer thickness being different for the strained QW and strained barrier structures.

The actual value of the relevant broadening parameter $\gamma$ is sensitive to all the processes leading to the structurally induced tails in the interband absorption but is not identical to the energy width of the absorbtion tail since it relies on the contribution of the lh-e transitions to the absorption at the hh-e absorption edge. Therefore, the broadening should also depend on the lh- hh- splitting. A large separation of the hh1- and lh1- valence band states in the strained-well SLs make them the most advantageous for polarized photoemission.

Then, the broadening effects may not be strong enough to account for large polarization losses observed in some of the strained SL structures [11, 28, 31] within a limited variation of the SL parameters. Additional polarization losses can be caused by the mixing between lh- and hh- states 
at the interfaces due to the lowered symmetry of the interface. For the case of ideally perfect interfaces discussed above this mixing does not affect the maximum polarization value in the first polarization maximum due to the left- and right-interface compensation. In the case of a real interface and allowing fluctuations of the alloy composition in the interface plane, the leftand right- interface contributions to the hh-lh mixing will not compensate each other. These effects are of a particular importance in the structures with "no common anion" heterointerfaces with enlarged value of the mixing parameter $t$ [22, 24].

Finally, the polarization of the electrons emitted in vacuum is also reduced in transport to the surface and emission through the surface band bending region and the activation layer. Time and energy-resolved experimental studies of the polarized photoemission give the estimates of these effects. Though the polarization losses in transport are not dominating, the mechanisms and relative contributions of the depolarization at these stages are still under debates [8]. The set of wavefunctions and the SL band spectra obtained here are prerequisites for calculations of the transport and spin relaxation parameters.

\section{Conclusions}

To summarize, a theoretical investigation of the electronic optical orientation in the unstrained, well-strained and the barrier-strained superlattices showed the dominating effect of the valence band splitting on the maximum polarization that can be achieved in the moment of excitation. The maximum splitting is found in the strained well superlattices, while the splitting in strain-barrier and strain-compensated structures with realistic parameters is lower that the deformation induced splitting. However, the achievable splitting values in these SLs can still be larger than in structures with one strained layer due to reduced strain relaxation effects.

The highest value of the polarization in the excitation moment is obtained for the structures where a high strain valence band splitting in the QW layers is accompanied by a large confinement splitting due to high barriers in the valence band. Technological limitations for the growth of the highly strained SL structures set restrictions to the realistic choice of the structures parameters, necessitating optimization of the photoemitter structure as a whole within these limitations. 


\section{Acknowledgments}

This work was supported by CRDF under grant RP1-2345-ST-02, SNSF under grant 7IP 062585 and RFBR under grant 00-02-16775. We are grateful to J.E. Clendenin for reading the manuscript.

\section{Appendix}

Matrixes $G$ and $\Gamma$ in the effective Hamiltonian (3) are defined e.g. in Ref. [15].

$$
\begin{aligned}
& \Gamma=-\frac{\Delta}{3}\left(\begin{array}{cccc}
0 & 0 & 0 & 0 \\
0 & 0 & 0 & -1 \\
0 & 0 & 0 & i \\
0 & 1 & -i & 0
\end{array}\right) \\
& G=\left(\begin{array}{cccc}
F_{c} & i P k_{x} & i P k_{y} & \frac{i}{2}\left(P \hat{k}_{z}+\hat{k}_{z} P\right) \\
-i P k_{x} & F_{x} & N k_{x} k_{y}-i \frac{\Delta}{3} & \frac{1}{2} k_{x}\left(N \hat{k}_{z}+\hat{k}_{z} N\right) \\
-i P k_{y} & N k_{x} k_{y}+i \frac{\Delta}{3} & F_{y} & \frac{1}{2} k_{y}\left(N \hat{k}_{z}+\hat{k}_{z} N\right) \\
-\frac{i}{2}\left(P \hat{k}_{z}+\hat{k}_{z} P\right) & \frac{1}{2} k_{x}\left(N \hat{k}_{z}+\hat{k}_{z} N\right) & \frac{1}{2} k_{y}\left(N \hat{k}_{z}+\hat{k}_{z} N\right) & F_{z}
\end{array}\right)
\end{aligned}
$$

Here $\hat{k}_{z}=-i \partial / \partial z, k_{x}$ and $k_{y}$ are components of the momentum $\mathbf{k}$,

$$
\begin{gathered}
F_{c}=E_{c}+A\left(k_{y}^{2}+k_{x}^{2}\right)+\hat{k}_{z} A \hat{k}_{z} \\
F_{x}=E_{v}+L k_{x}^{2}+M k_{y}^{2}+\hat{k}_{z} M \hat{k}_{z} \\
F_{y}=E_{v}+L k_{y}^{2}+M k_{x}^{2}+\hat{k}_{z} M \hat{k}_{z} \\
F_{z}=E_{v}+\hat{k}_{z} L \hat{k}_{z}+M\left(k_{y}^{2}+k_{x}^{2}\right)
\end{gathered}
$$

Inside the SL layers 1 and 2 all parameters in (1617) $E_{c, v}, \Delta, P, A, L, M, N$ are constant and equal to the corresponding values of the bulk materials. Therefore the order of the operators $\hat{k}_{z}$ and Hamiltonian parameters is not essential. However in the interface region where parameters are considered as step functions this order becomes important. We use the symmetrized form following [14]. 


\section{References}

[1] K. Abe et al. Phys. Rev. Lett. 861162 (2001).

[2] M. Poelker, P. Adderley, J. Clark, A. Day, J. Grames, C. Sinclair et al., Proc 14th Intern Spin Physics Symp. (SPIN 2000) (AIP Conf. Proc. Vol. 570, Eds. K. Hatanaka et al., Melville, NY) p. 698.

[3] K. Aulenbacher, Ch. Nachtigall, H.G. Andersen, P. Dresher, H. Euteneur, H. Fischer, D. v. Harrach, P. Hartmann, J. Hoffman, P. Jennewein, K.-H. Kaiser, H. J. Kreidel, S.Plutzer, E. Reichert, K.-H. Steffens, and M. Steigerwald, Nucl. Instrum. and Methods Phys. Res. A 391, 498 (1997).

[4] Proc 15th Intern Spin Physics Symp.(SPIN 2002) (AIP Conf. Proc. Vol. 570, Eds. K. Hatanaka et al., Melville, NY) p. 698.

[5] Optical orientation, edited by F.Meier and B.P.Zakharchenya, NorthHolland, 1984.

[6] R.A.Mair, R. Prepost, E.L. Garvin, and T.Maruyama, Phys. Lett. A, 239, 277 (1998).

[7] A.V. Subashiev, Yu.A. Mamaev, B.D. Oskotskii, Yu.P. Yashin, and V.K. Kalevich, Semiconductors 33, 1182 (1999).

[8] K. Aulenbacher, J. Schuler, D. v. Harrach, E. Reichert, J. Roethgen, A. Subashiev, V. Tioukine, and Y. Yashin, J. Appl. Phys. 92, 7536 (2002).

[9] A.D. Andreev, and A. V. Subashiev, Physica E, 13, 556 (2002).

[10] A.V. Subashiev, A.V., Mamaev, Yu.A., Yashin, Yu.P., Clendenin, J.E., Phys. Low-Dim. Structures, 1/2, 1 (1999).

[11] T. Nakanishi, H. Aoyagi, T. Kosugoh, S. Nakamura, M.Tawada, H. Horinaka, and Y. Kamiya, Surface Science, 454-456, 1042 (2000).

[12] A. Twardowski, C. Hermann, Phys. Rev. B 43, 8144 (1987).

[13] I.A. Merkulov, V.I. Perel', M.E. Portnoi, Zh. Exp. Teor. Fiz. 99, 1202 (1990) [Sov. Phys. JETP 72, 669 (1991)]. 
[14] G.A. Baraff and D. Gershoni, Phys. Rev. B 43, 4011 (1991), D. Gershoni, C. H. Henry and G.A. Baraf, IEEE Journ. Quant. Electron., 29 2433 (1993).

[15] E. O. Kane, "Energy Band Theory", in Handbook on Semiconductors, vol. 1, ed. W. Paul, North-Holland, 1982. pp. 193-217.

[16] I. Vurgaftman, J. R. Meyer and L.R. Ram-Mohan, J. Appl. Phys. 89, 5815 (2001).

[17] D. L. Smith and C. Mailhiot, Rev. Modern Phys., 62173 (1990).

[18] E. L. Ivchenko and G.E. Pikus, Superlattices and Other Heterostructures (Springer, Berlin, 1995).

[19] A. V. Rodina, A. Yu. Alekseev, Al. L. Efros, M. Rosen and B.K. Meyer, Phys. Rev. B 65, 125302-1 (2002).

[20] E.L. Ivchenko, A.Yu. Kaminski, and I.L. Aleiner, Zh. Exp. Teor Fiz. 104, 3401 (1993) [JETP 77, 609 (1993)].

[21] E.L. Ivchenko, A.Yu. Kaminski, and U. Rössler, Phys. Rev. B 54, 5852 (1996).

[22] L. Vervoort, F Ferreira, P. Voisin, Phys. Rev. B 56, R12 744 (1997).

[23] L.E. Golub, Phys. Rev, B 56, R12 744 (1997).

[24] E.L. Ivchenko, A.A. Toropov, and P. Voisin, Fiz. Tver. Tela 40, 1925 (1998) [Solid State Physics, 77, 609 (1993)].

[25] Excitonic effects in the absorption spectra at the excitation edges are not included since they do not give noticeable cobntribution to the photoemission current and no signes of these effects in photoemission were observed experimentally for the presence.

[26] F. Szmulowicz, Phys. Rev. B 511613 (1995).

[27] C.W. Greef, H. R. Glyde, Phys. Rev. B 51, 1778 (1995).

[28] T. Maruyama, Proc. of the 14-th Intern. Symp. on High-Energy Spin Physics, (SPIN 2002) (AIP, Melville, New York, 2003) 1029. 
[29] A. V. Subashiev, Yu. A. Mamaev, Yu. P.Yashin, V. M. Ustinov, A. E. Zhukov, J. E. Clendenin, T. Maruyama, G. A. Mulhollan, SLACPub 7922, 1998, also in Proc. of the 24-th Intern. Conf. on Physics of Semicond, (ICPS-24).

[30] A. N. Ambrajei, J. E. Clendenin, A. Yu. Egorov, Yu. A. Mamaev, T. Maruyama, G. A. Mulhollan, A. V. Subashiev, Yu. P. Yashin, V. M. Ustinov, A. E. Zhukov, Appl. Surface Science, 166, 40 (2000).

[31] Yu. P. Yashin, J. S. Roberts, L. G. Gerchikov, P. A. Houston, A. N. Ipatov, Yu. A. Mamaev, A. V. Rochansky and A. V. Subashiev 11th Int. Symp. "Nanostructures: Physics and Technology" (NANO 2003) (St.Petersburg, 2003) p. 61. 